\title{
Walking Speed: Japanese Data in Chronic Liver Diseases
}

\author{
Hiroki Nishikawa ${ }^{1,2, *}$, Hirayuki Enomoto ${ }^{1}$, Kazunori Yoh ${ }^{1}$, Yoshinori Iwata ${ }^{1}$, \\ Yoshiyuki Sakai ${ }^{1}$, Kyohei Kishino ${ }^{1}$, Naoto Ikeda ${ }^{1}$, Tomoyuki Takashima ${ }^{1}$, Nobuhiro Aizawa ${ }^{1}$, \\ Ryo Takata ${ }^{1}$, Kunihiro Hasegawa ${ }^{1}$, Noriko Ishii ${ }^{1}$, Yukihisa Yuri ${ }^{1}$, Takashi Nishimura ${ }^{1}$, \\ Hiroko Iijima ${ }^{1}$ and Shuhei Nishiguchi ${ }^{1}$ \\ 1 Division of Hepatobiliary and Pancreatic Disease, Department of Internal Medicine, Hyogo College of \\ Medicine, Nishinomiya, Hyogo 663-8501, Japan; enomoto@hyo-med.ac.jp (H.E.); \\ mm2wintwin@ybb.ne.jp (K.Y.); yo-iwata@hyo-med.ac.jp (Y.I.); sakai429@hyo-med.ac.jp (Y.S.); \\ hcm.kyohei@gmail.com (K.K.); nikeneko@hyo-med.ac.jp (N.I.); tomo0204@yahoo.co.jp (T.T.); \\ nobu23hiro@yahoo.co.jp (N.A.); chano_chano_rt@yahoo.co.jp (R.T.); hiro.red1230@gmail.com (K.H.); \\ ishinori1985@yahoo.co.jp (N.I.); gyma27ijo04td@gmail.com (Y.Y.); tk-nishimura@hyo-med.ac.jp (T.N.); \\ hiroko-i@hyo-med.ac.jp (H.I.); nishiguc@hyo-med.ac.jp (S.N.) \\ 2 Center for Clinical Research and Education, Hyogo College of Medicine, Nishinomiya \\ Hyogo 663-8501, Japan \\ * Correspondence: nishikawa_6392_0207@yahoo.co.jp; Tel.: +81-798-45-6111; Fax: +81-798-45-6608
}

Received: 30 November 2019; Accepted: 3 January 2020; Published: 7 January 2020

\begin{abstract}
We aim to clarify the impact of walking speed (WS) and analyze factors linked to WS decline in patients with chronic liver diseases (CLDs, 165 males and 191 females, 137 liver cirrhosis patients). The WS decline is defined as $<0.8 \mathrm{~m} / \mathrm{second}(\mathrm{m} / \mathrm{s})$, referring to the guidelines. The median (range) WS was $1.3 \mathrm{~m} / \mathrm{s}(0.2-2.02 \mathrm{~m} / \mathrm{s})$. There were 17 patients with $\mathrm{WS}<0.8 \mathrm{~m} / \mathrm{s}(4.8 \%)$. The WS value was significantly correlated with the handgrip strength value both in males $\left(r^{2}=0.252, p<0.0001\right)$ and females $\left(r^{2}=0.256, p<0.0001\right)$. In the multivariate analysis of factors associated with WS decline, only the extracellular water (ECW) to total body water (TBW) ratio using bioimpedance analysis was an independent predictor $(p=0.0398)$. Extracellular fluid excess was categorized as follows: normal condition (ECW to TBW ratio < 0.390), mild overhydrated condition (ECW to TBW ratio 0.390-0.399), and moderate to severe overhydrated condition (ECW to TBW ratio $\geq 0.400$ ). The WS value was well stratified according to the ECW to TBW ratio (normal vs. mild, $p=0.0001$; mild vs. moderate to severe, $p<0.0001$; normal vs. moderate to severe, $p<0.0001$; overall $p$-value $<0.0001$ ). In conclusion, the ECW to TBW ratio can be closely linked to WS decline in CLD patients.
\end{abstract}

Keywords: walking speed; handgrip strength; chronic liver diseases; ECW to TBW ratio; sarcopenia

\section{Introduction}

Because sarcopenia, as defined by muscle mass decline and muscle strength decline in patients with chronic liver diseases (CLDs), can be associated with falls, decreased quality of life, or poor prognosis, it has gained much interest among clinicians these days [1-7]. Sarcopenia, especially, can occur frequently in patients with liver cirrhosis (LC), and it can be a risk factor for the development of hepatic encephalopathy in LC patients [8,9]. Japanese CLD patients are aging these days, and it is also an important public health problem as aging can be associated with sarcopenia $[10,11]$. How sarcopenia is associated with such adverse clinical outcomes needs to be looked at beyond the overt muscle mass loss or muscle strength decline and at this clinical entity as a systemic disease [12-14]. Muscle mass has been assessed by different techniques such as the bioimpedance analysis (BIA), dual-energy X-ray, computed tomography (CT) and magnetic resonance imaging, and the functional 
consequence of sarcopenia is estimable by handgrip strength (HGS) or other more composite tests such as the walking test [10,15-17]. Physical activity, nutrition, and control of underlying diseases may be required for the prevention of sarcopenia [5].

Walking speed (WS) can reflect skeletal muscle function and is an easy and reproducible measure [18-20]. Currently, the European Working Group on Sarcopenia in Older People (EWGSOP) criteria and the Asian Working Group on Sarcopenia (AWGS) criteria define $0.8 \mathrm{~m} / \mathrm{s}$ as the cutoff point for WS decline $[15,16]$. However, in a population-based, cross-sectional survey of Japanese adults aged 65 years or older who were living independently at home in the community, the proportion of adults presenting a WS of $<0.8 \mathrm{~m} / \mathrm{s}$ was only $3.6 \%$ (174/4811) [21]. The WS, thus, may not be suitable for the screening tool of muscle strength decline in Japanese older adults. Moreover, since clear consensus has not been reached with regard to assessment methods for WS (e.g., 12-, 10-, 8-, 6- meter walking), the Japanese Society of Hepatology (JSH) adopts only the HGS as a marker of muscle strength, and WS is not included in the current JSH guidelines for sarcopenia in CLDs [10]. Based on these backgrounds, there have been few reports examining the impact of WS in Japanese CLD patients [18,19]. In addition, recent research reported that in adults aged $\geq 60$ years living in the community, WS decline and the HGS decline are associated with cognitive decline but there may be different mechanisms between brain and physical functions [20]. There is, thus, an urgent need for clarifying the impact of WS in CLDs, and in this study we seek to address these issues.

\section{Patients and Methods}

\subsection{Patients}

A total of 356 CLD individuals with data for WS were admitted to our hospital between July 2015 and January 2019, and they were subjected to this analysis. HGS data were available in all analyzed subjects, which were tested referring to the Japanese guidelines [10]. Presence of LC was judged by pathological findings (F4 or not), radiological findings (deformity of the liver, or the presence of varices or splenomegaly), and/or laboratory data (lower platelet count or lower prothrombin time) [22-25]. Mac-2 binding protein glycosylation isomer (M2BPGi) as a liver fibrosis marker was tested, as reported elsewhere [26]. Skeletal muscle index $\left(\mathrm{SMI}, \mathrm{kg} / \mathrm{m}^{2}\right)$ was tested using BIA, as reported elsewhere [27]. SMI decline was defined as $<7.0 \mathrm{~kg} / \mathrm{m}^{2}$ in males and $<5.7 \mathrm{~kg} / \mathrm{m}^{2}$ in females, referring to the Japanese guidelines [10]. Two patients had missing data for BIA. Severe ascites patients were not subject to this analysis owing to the limitation of BIA testing. We examined the relationship between the baseline of the WS value and clinical variables in a retrospective manner. WS decline was defined as $<0.8 \mathrm{~m} / \mathrm{s}$ referring to the current EWGSOP or AWGS guidelines $[15,16]$. Factors related to the WS decline were also explored in the univariate and multivariate analyses.

The current research protocol was approved by the institutional review board (IRB) of the Hyogo College of Medicine Hospital, and the declaration of Helsinki was strictly followed to ensure the rights of the research subjects (IRB approval no. 2296). Personal information was protected in the data collection.

\subsection{Measurement of Walking Speed}

In this study, a 6-m walking test was performed in each analyzed subject. We performed 6-m walking tests twice in each patient and adopted the average value of them as the walking speed (meter/second $(\mathrm{m} / \mathrm{s}))$.

\subsection{Statistical Considerations}

The JMP 14 software (SAS Institute Inc., Cary, NC, USA) was in use to do statistical analysis.

For the continuous parameter analysis, Student's $t$-test, the Mann-Whitney U-test or Pearson's correlation coefficient $r$, analysis of variance or the Kruskal-Wallis test was in use to adequately evaluate group differences. For the categorical parameter analysis, Fisher's exact test or Pearson $\chi^{2}$ test was in 
use to evaluate group differences. Baseline items with significant correlation with the WS decline in our univariate analysis were subject to multivariate logistic regression analysis to choose candidate items. Unless otherwise mentioned, data were shown as the median value (range). The statistical significance threshold was set at $p<0.05$.

\section{Results}

\subsection{Baseline Features}

Baseline features of the analyzed subjects $(n=356)$ are presented in Table 1 . The study cohort included 165 males and 191 females with the median age (range) of 66 (25-94) years. In terms of the disease etiology for CLD, hepatitis C virus was in the majority (181/356, 50.8\%). The median (range) WS was $1.3 \mathrm{~m} / \mathrm{s}(0.2-2.02 \mathrm{~m} / \mathrm{s})$. Number distribution for the WS value is demonstrated in Figure 1A. The number of patients with $1.2 \mathrm{~m} / \mathrm{s}<\mathrm{WS}<1.4 \mathrm{~m} / \mathrm{s}$ was the highest $(110$ cases, $30.9 \%$ ). LC was identified at baseline in 137 cases (38.5\%). There were 17 patients with WS $<0.8 \mathrm{~m} / \mathrm{s}(4.8 \%)$ and 51 patients with WS $<1.0 \mathrm{~m} / \mathrm{s}(14.3 \%)$. In males, the median (range) HGS was $33.6 \mathrm{~kg}(13.6-53.6 \mathrm{~kg})$, while in females, the median (range) HGS was $20.5 \mathrm{~kg}(6.0-34.8 \mathrm{~kg})$. Twenty-eight males $(17.0 \%)$ and 57 females $(29.8 \%)$ had the HGS decrease (HGS: $<26 \mathrm{~kg}$ or $<18 \mathrm{~kg}$ ). In males, the median (range) SMI was $7.475 \mathrm{~kg} / \mathrm{m}^{2}$ $\left(5.21-11.01 \mathrm{~kg} / \mathrm{m}^{2}\right)$, while in females, the median (range) SMI was $5.875 \mathrm{~kg} / \mathrm{m}^{2}\left(3.90-8.17 \mathrm{~kg} / \mathrm{m}^{2}\right)$. The median (range) WS in LC patients $(1.23 \mathrm{~m} / \mathrm{s}(0.33-1.76 \mathrm{~m} / \mathrm{s})$ was significantly slower than that in non-LC patients $(1.36 \mathrm{~m} / \mathrm{s}(0.2-2.02 \mathrm{~m} / \mathrm{s}) ; p<0.0001$; Figure 1B). The difference of WS between patients aged $<65$ years $(n=162)$ and those aged $\geq 65$ years $(n=194)$ also reached significance $(p<0.0001$; median (range): $1.365 \mathrm{~m} / \mathrm{s}(0.33-1.85 \mathrm{~m} / \mathrm{s})$ vs. $1.22 \mathrm{~m} / \mathrm{s}(0.20-2.02 \mathrm{~m} / \mathrm{s})$; Figure $2 \mathrm{~A})$ The difference of WS between male patients and female patients did not reach significance $(p=0.5577$; median (range): $1.28 \mathrm{~m} / \mathrm{s}(0.2-2.02 \mathrm{~m} / \mathrm{s})$ vs. $1.31 \mathrm{~m} / \mathrm{s}(0.33-1.84 \mathrm{~m} / \mathrm{s})$; Figure $2 \mathrm{~B})$.

Table 1. Baseline characteristics $(n=356)$.

\begin{tabular}{cc}
\hline Variables & $\begin{array}{c}\text { Number or } \\
\text { Median Value (Range) }\end{array}$ \\
\hline Age (years) & $66(25,94)$ \\
Gender, male/female & $165 / 191$ \\
HCV/HBV/HCV and HBV/NBNC & $181 / 61 / 9 / 105$ \\
Body mass index $\left(\mathrm{kg} / \mathrm{m}^{2}\right)$ & $22.7(14.6,41.4)$ \\
Walking speed $(\mathrm{m} / \mathrm{s})$ & $1.3(0.2,2.02)$ \\
Hand grip strength $(\mathrm{kg}$, male $)$ & $33.6(13.6,53.6)$ \\
Hand grip strength $(\mathrm{kg}$, female) & $20.5(6.0,34.8)$ \\
Presence of LC, yes/no & $137 / 219$ \\
Total bilirubin $(\mathrm{mg} / \mathrm{dL})$ & $0.8(0.2,5.1)$ \\
Serum albumin $(\mathrm{g} / \mathrm{dL})$ & $4.3(2.0,5.2)$ \\
Prothrombin time $(\%)$ & $91.2(11.9,122.9)$ \\
Platelet count $\left(\times 10^{4} / \mathrm{mm}^{3}\right)$ & $17.5(1.4,51.4)$ \\
FIB-4 index & $2.16(0.29,30.98)$ \\
M2BPGi $(\mathrm{cutoff}$ index $)$ & $1.255(0.21,11.93)$ \\
SMI $\left(\mathrm{kg} / \mathrm{m}^{2}, \mathrm{male}\right)$ & $7.475(5.21,11.01)$ \\
SMI $\left(\mathrm{kg} / \mathrm{m}^{2}\right.$, female $)$ & $5.875(3.90,8.17)$ \\
ECW to TBW ratio & $0.389(0.367,0.425)$ \\
AST $(\mathrm{IU} / \mathrm{L})$ & $25(10,222)$ \\
ALT $(\mathrm{IU} / \mathrm{L})$ & $19(5,232)$ \\
HbA1c $(\mathrm{NGSP})$ & $5.7(3.7,12.6)$ \\
eGFR $\left(\mathrm{ml} / \mathrm{min}^{2} / .73 \mathrm{~m}{ }^{2}\right)$ & $81(5,173)$ \\
Serum zinc $(\mu \mathrm{g} / \mathrm{dL})$ & $73.9(22.0,124.6)$ \\
BTR & $5.65(1.69,13.82)$ \\
Serum ammonia $(\mu \mathrm{gg} / \mathrm{dL})$ & $38(12,206)$ \\
\hline
\end{tabular}

HCV: hepatitis C virus, HBV: hepatitis B virus, NBNC: non-B and non-C, HCC, LC: liver cirrhosis, M2BPGi: Mac-2 binding protein glycosylation isomer, SMI: skeletal muscle index, ECW: extracellular water, TBW: total body water, AST: aspartate aminotransferase, ALT: alanine aminotransferase, NGSP: National Glycohemoglobin Standardization Program, eGFR: estimated glomerular filtration rate, BTR: branched-chain amino acid to tyrosine ratio. 
(A)
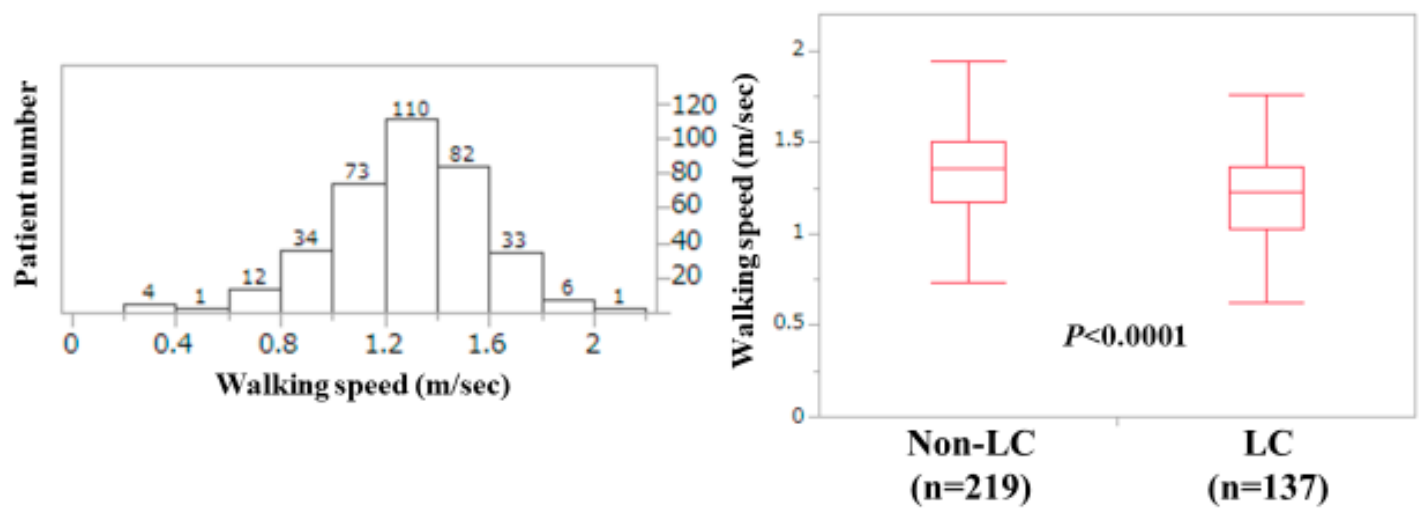

(B)

Figure 1. (A) Patient number distribution according to walking speed (WS). (B) The WS value according to the LC status.

(A)

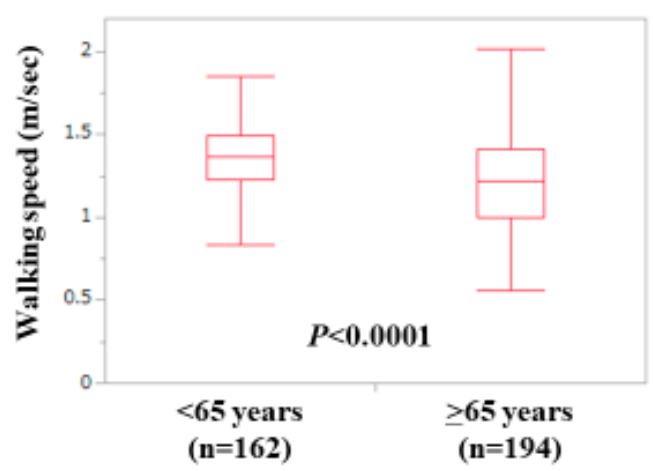

(B)

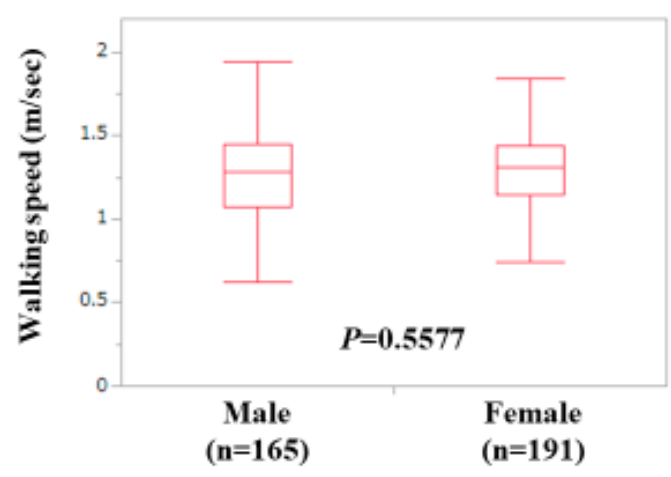

Figure 2. (A) The WS value according to age. (B) The WS value according to gender.

\subsection{Relationship between WS and HGS for All Cases, LC Cases and Non-LC Cases}

For all cases, the WS value was significantly correlated with the HGS value both in males $\left(r^{2}=0.252\right.$, $p<0.0001)$ and females $\left(r^{2}=0.256, p<0.0001\right.$; Figure 3A,B). For LC cases, the correlation between the WS value and the HGS value reached significance in both males $\left(r^{2}=0.134, p=0.0019\right)$ and females $\left(r^{2}=0.156, p=0.0009\right.$; Figure 4A,B). For non-LC cases, the correlation between the WS value and the HGS value reached significance in both males $\left(r^{2}=0.277, p<0.0001\right)$ and females $\left(r^{2}=0.269, p=0.0009\right.$; Figure $4 \mathrm{C}, \mathrm{D}$ ). In male patients, the proportion of patients with both HGS $<26 \mathrm{~kg}$ (cutoff value in the JSH guidelines) and WS $<0.8 \mathrm{~m} / \mathrm{s}$ was $3.0 \%(5 / 165)$, while that with both HGS $<26 \mathrm{~kg}$ and WS $<1.0 \mathrm{~m} / \mathrm{s}$ was $6.7 \%(11 / 165)$. In female patients, the proportion of patients with both HGS $<18 \mathrm{~kg}$ (cutoff value in the JSH guidelines) and WS $<0.8 \mathrm{~m} / \mathrm{s}$ was $2.6 \%(5 / 191)$, while that with both HGS $<18 \mathrm{~kg}$ and WS $<1.0 \mathrm{~m} / \mathrm{s}$ was $8.9 \%(17 / 191)[10]$. 
(A)

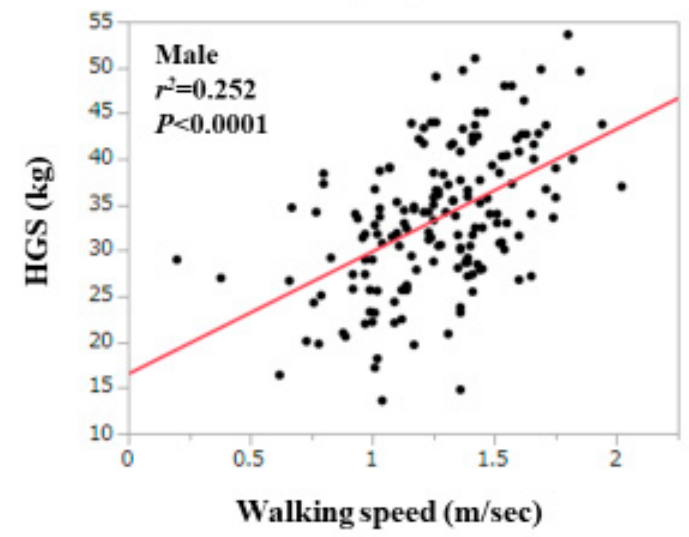

(B)

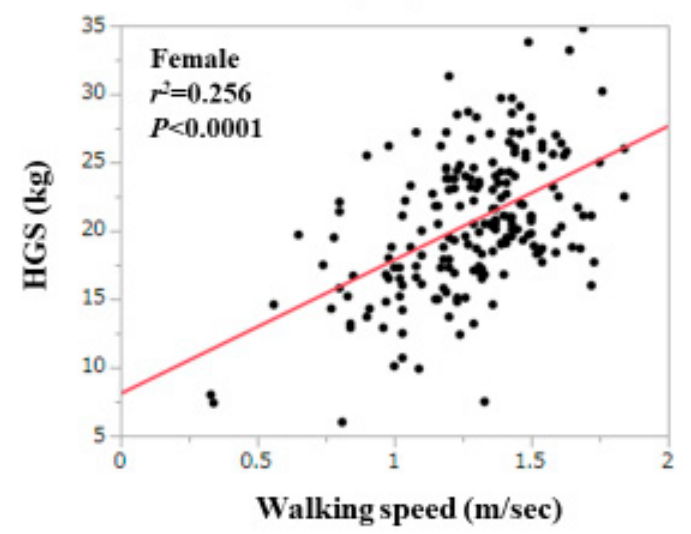

Figure 3. Correlation between the WS value and the handgrip strength value in males (A) and in females (B).
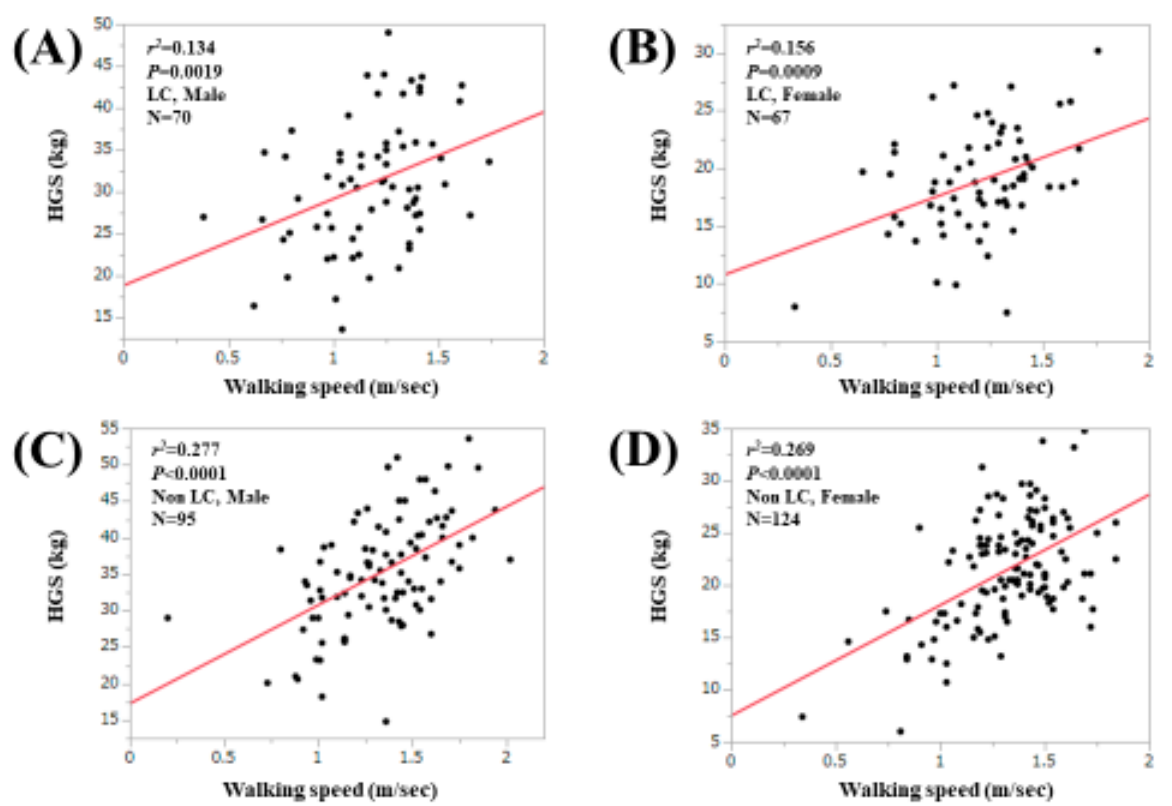

Figure 4. Correlation between the WS value and the handgrip strength value in male LC patients (A) and in female LC patients (B). Correlation between the WS value and the handgrip strength value in male non-LC patients (C) and in female non- LC patients (D).

\subsection{Relationship between WS and SMI for All Cases, LC Cases and Non-LC Cases}

For all cases, the WS value was significantly correlated with the SMI value only in males $\left(r^{2}=0.039\right.$, $p=0.0111$; Figure 5A,B). For LC cases, the correlation between the WS value and the HGS value did not reach significance in both males $\left(r^{2}=0.024, p=0.1983\right)$ and females $\left(r^{2}=0.013, p=0.3546\right.$; Figure 6A,B). For non-LC cases, the correlation between the WS value and the HGS value reached significance only in males $\left(r^{2}=0.097, p=0.0022\right.$; Figure $\left.6 \mathrm{C}, \mathrm{D}\right)$. 
(A)

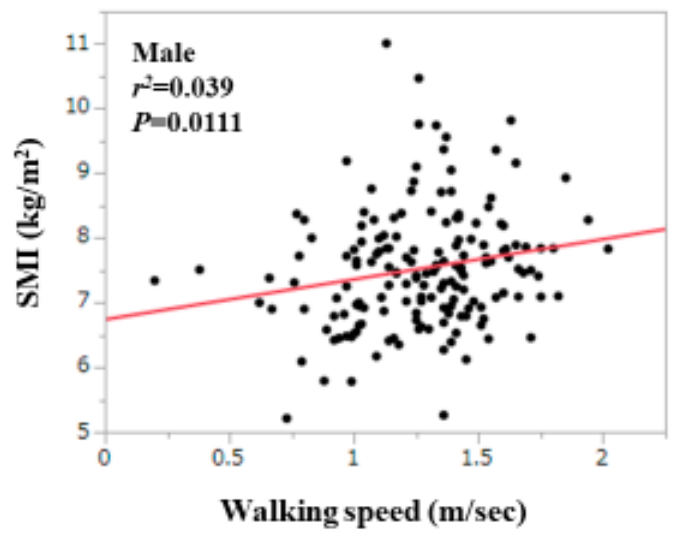

(B)

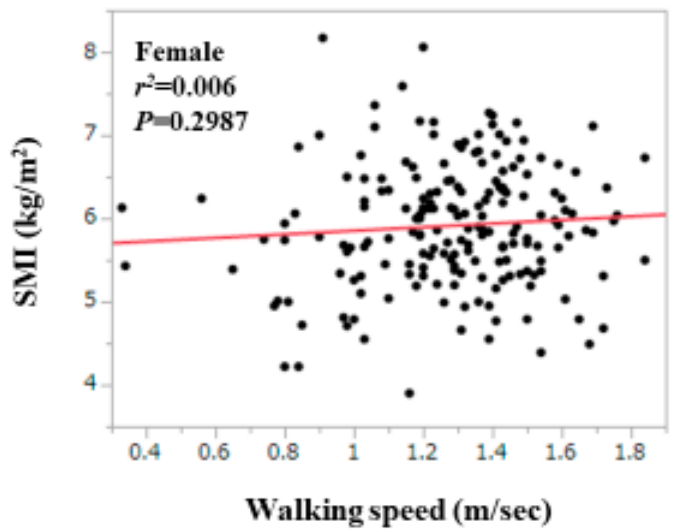

Figure 5. Correlation between the WS value and the SMI value in males (A) and in females (B).

(A)

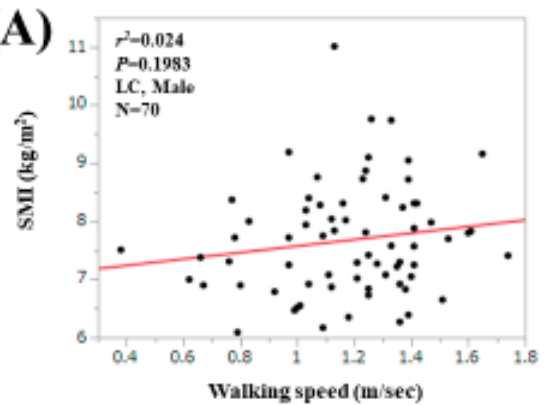

(C)

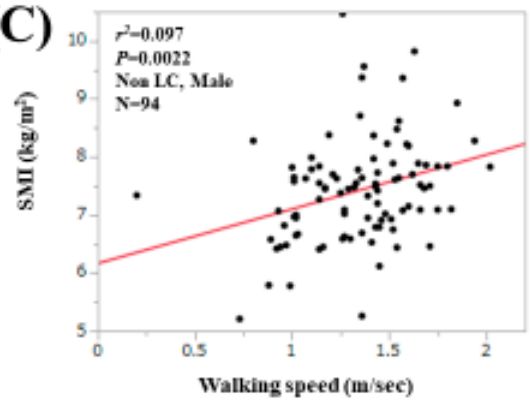

(B)

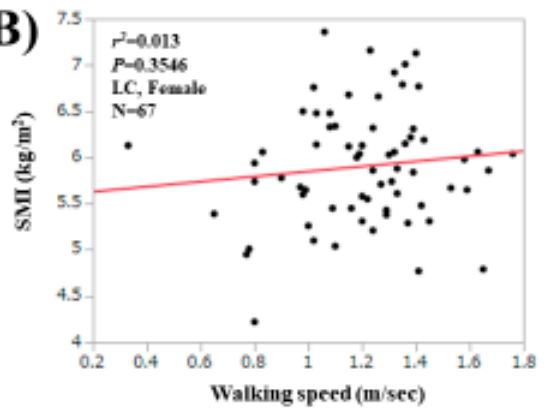

(D)

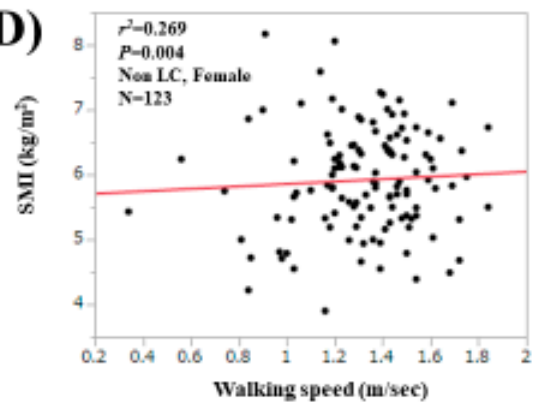

Figure 6. Correlation between the WS value and the SMI value in male LC patients (A) and in female LC patients (B). Correlation between the WS value and the SMI value in male non-LC patients (C) and in female non-LC patients (D).

\subsection{Correlation Coefficients between WS and Baseline Parameters Other than HGS}

Correlation coefficients between WS and baseline parameters other than HGS are shown in Table 2. Among these, serum albumin involved the strongest positive correlation with WS $(r=0.309146$, $p<0.0001$ ), while the extracellular water (ECW) to total body water (TBW) ratio, as assessed by BIA, involved the strongest negative correlation with WS $(r=-0.41169, p<0.0001)$. 
Table 2. Correlation between walking speed and baseline data other than handgrip strength.

\begin{tabular}{ccc}
\hline & $r$ & $p$-Value \\
\hline Age & -0.3002 & $<0.0001$ \\
Body mass index & -0.02932 & 0.5825 \\
Total bilirubin & -0.07285 & 0.1702 \\
Serum albumin & 0.309146 & $<0.0001$ \\
Prothrombin time & 0.142872 & 0.0069 \\
Platelet count & 0.091789 & 0.0837 \\
AST & -0.06229 & 0.2411 \\
ALT & 0.02578 & 0.6278 \\
FIB-4 index & -0.17981 & 0.0007 \\
HbA1c (NGSP) & -0.05046 & 0.3459 \\
eGFR & 0.090435 & 0.0884 \\
SMI, male & 0.189214 & 0.0149 \\
SMI, female & 0.091065 & 0.2127 \\
ECW to TBW ratio & -0.41169 & $<0.0001$ \\
BTR & 0.252886 & $<0.0001$ \\
Serum zinc & 0.195912 & 0.0011 \\
Serum ammonia & -0.16623 & 0.0039 \\
M2BPGi & -0.2555 & $<0.0001$
\end{tabular}

AST, aspartate aminotransferase; ALT, alanine aminotransferase; NGSP, National Glycohemoglobin Standardization Program; eGFR, estimated glomerular filtration rate; SMI, skeletal muscle index; ECW, extracellular water; TBW, total body water; BTR, branched-chain amino acid to tyrosine ratio; M2BPGi, Mac-2 binding protein glycosylation isomer.

\subsection{Uni- and Multivariate Analyses of Factors Related to the WS Decline ( $<0.8 \mathrm{~m} / \mathrm{s})$}

The univariate analysis found six variables to have significant association with WS decline $(<0.8 \mathrm{~m} / \mathrm{s})$ : age $(p=0.0278)$, presence of LC $(p=0.0088)$, serum albumin level $(p=0.0246)$, M2BPGi $(p=0.0141)$, branched-chain amino acid to tyrosine ratio (BTR; $p=0.0127)$ and ECW to TBW ratio $(p<0.0001$; Table 3). Multivariate analysis for the six factors showed that only the ECW to TBW ratio $(p=0.0398)$ was a significant factor linked to WS decline $(<0.8 \mathrm{~m} / \mathrm{s}$; Table 4$)$. Hazard ratios (HRs) and 95\% confidence intervals (CIs) are shown in Table 4.

Table 3. Univariate analyses of factors linked to walking speed (WS) decline $(<0.8 \mathrm{~m} / \mathrm{s})$.

\begin{tabular}{|c|c|c|c|}
\hline Variables & $\begin{array}{c}\mathrm{WS} \geq 0.8 \mathrm{~m} / \mathrm{s} \\
(\mathrm{n}=339)\end{array}$ & $\begin{array}{c}\text { WS }<0.8 \mathrm{~m} / \mathrm{s} \\
\quad(\mathrm{n}=17)\end{array}$ & $p$-Value \\
\hline Age (years) & $65(29,94)$ & $71(25,83)$ & 0.0278 \\
\hline Gender, male/female & $155 / 184$ & $10 / 7$ & 0.3265 \\
\hline $\mathrm{HBV} / \mathrm{HCV} / \mathrm{HBV}$ and $\mathrm{HCV} / \mathrm{NBNC}$ & 60/170/9/100 & $1 / 11 / 0 / 5$ & 0.4852 \\
\hline Body mass index $\left(\mathrm{kg} / \mathrm{m}^{2}\right)$ & $22.7(14.6,41.4)$ & $21.5(17.7,29.2)$ & 0.3367 \\
\hline Presence of LC, yes/no & $125 / 214$ & $12 / 5$ & 0.0088 \\
\hline Total bilirubin (mg/dL) & $0.8(0.2,5.1)$ & $0.9(0.4,3.2)$ & 0.7231 \\
\hline Serum albumin $(\mathrm{g} / \mathrm{dL})$ & $4.3(2.0,5.2)$ & $4.0(2.4,4.6)$ & 0.0246 \\
\hline Prothrombin time (\%) & $91.4(11.9,122.9)$ & $84.7(46.5,103.9)$ & 0.1597 \\
\hline Platelet count $\left(\times 10^{4} / \mathrm{mm}^{3}\right)$ & $17.7(1.4,51.4)$ & $16.0(2.8,32.2)$ & 0.1401 \\
\hline AST & $25(12,222)$ & $27(10,191)$ & 0.5570 \\
\hline ALT & $19(5,206)$ & $16(5,232)$ & 0.2200 \\
\hline M2BPGi & $1.24(0.21,11.93)$ & $2.31(1.08,10.82)$ & 0.0141 \\
\hline eGFR $\left(\mathrm{ml} / \mathrm{min} / 1.73 \mathrm{~m}^{2}\right)$ & $81(5,173)$ & $76(7,99)$ & 0.0931 \\
\hline HbA1c (NGSP) & $5.7(3.7,12.6)$ & $5.7(4.6,7.2)$ & 0.3573 \\
\hline Serum ammonia $(\mu \mathrm{g} / \mathrm{dL})$ & $38(12,195)$ & $48(18,206)$ & 0.0534 \\
\hline BTR & $5.71(1.93,13.82)$ & $3.92(1.69,8.8)$ & 0.0127 \\
\hline Serum zinc $(\mu \mathrm{g} / \mathrm{dL})$ & $73.9(22.0,124.6)$ & $68.4(38.0,94.4)$ & 0.0764 \\
\hline ECW to TBW ratio & $0.389(0.367,0.425)$ & $0.398(0.387,0.421)$ & $<0.0001$ \\
\hline SMI decline, yes/no/unknown & $114 / 223 / 2$ & $7 / 10 / 0$ & 0.6024 \\
\hline
\end{tabular}

Continuous data are expressed as median (range). $\mathrm{HCV}$, hepatitis $\mathrm{C}$ virus; $\mathrm{HBV}$, hepatitis $\mathrm{B}$ virus; NBNC, non-B and non-C; LC, liver cirrhosis; AST, aspartate aminotransferase; ALT, alanine aminotransferase; M2BPGi, Mac-2 binding protein glycosylation isomer; eGFR, estimated glomerular filtration rate; NGSP, National Glycohemoglobin Standardization Program; BTR, branched-chain amino acid to tyrosine ratio; ECW, extracellular water; TBW, total body water; SMI, skeletal muscle index. 
Table 4. Multivariate analyses of factors linked to the walking speed decline $(<0.8 \mathrm{~m} / \mathrm{s})$.

\begin{tabular}{cccc}
\hline & \multicolumn{3}{c}{ Multivariate Analysis } \\
\cline { 2 - 4 } & HR & $\mathbf{9 5 \%}$ CI & $p$-Value \\
\hline Age $^{\#}$ & 0.0267 & $9.251 \times 10^{-5}-7.700$ & 0.1594 \\
Presence of LC $^{\#}$ & 0.779 & $0.109-5.589$ & 0.8040 \\
Serum albumin $^{\#}$ & 15.152 & $0.0548-4184.100$ & 0.3333 \\
M2BPGi & 0.764 & $0.0117-49.763$ & 0.8998 \\
BTR $^{\#}$ & 8.007 & $0.0614-1044.325$ & 0.3874 \\
ECW to TBW ratio $^{\#}$ & 0.00105 & $1.387 \times 10^{-6}-0.795$ & 0.0398 \\
\hline
\end{tabular}

\footnotetext{
\# When a continuous variable changes over the entire range. HR, hazard ratio; CI, confidence interval; LC, liver cirrhosis; M2BPGi, Mac-2 binding protein glycosylation isomer; BTR, branched-chain amino acid to tyrosine ratio; ECW, extracellular water; TBW, total body water.
}

\subsection{Uni- and Multivariate Analyses of Factors Related to the WS Decline ( $<1.0 \mathrm{~m} / \mathrm{s})$}

The national center for geriatrics and gerontology in Japan (National Institute for Longevity Sciences-Longitudinal Study of Aging (NILS-LSA)) and the International Working Group on Sarcopenia (IWGS) define the optimal cutoff point of WS for muscle function decline as $<1.0 \mathrm{~m} / \mathrm{s}[17,28]$. Thus, uni- and multivariate analyses of factors related to WS decline $(<1.0 \mathrm{~m} / \mathrm{s})$ were also performed. The univariate analysis found seven factors to have significant association with WS decline $(<1.0 \mathrm{~m} / \mathrm{s})$ : age $(p<0.0001)$, presence of LC $(p=0.0124)$, serum albumin level $(p=0.0011)$, M2BPGi $(p=0.0016)$, BTR $(p=0.0289)$, ECW to TBW ratio $(p<0.0001)$, and presence of SMI decline ( $p=0.0037$; Table 5$)$. In the multivariate analysis for the seven factors, no significant factor was found. Age $(P=0.0995)$ and ECW to TBW $(P=0.0556)$ tended to be significant. HRs and 95\% CIs are shown in Table 6.

Table 5. Univariate analyses of factors linked to walking speed (WS) decline $(<1.0 \mathrm{~m} / \mathrm{s})$.

\begin{tabular}{|c|c|c|c|}
\hline Variables & $\begin{aligned} & W S \geq 1.0 \mathrm{~m} / \mathrm{s} \\
&(\mathrm{n}=305)\end{aligned}$ & $\begin{array}{c}\text { WS }<1.0 \mathrm{~m} / \mathrm{s} \\
(\mathrm{n}=51)\end{array}$ & $p$-Value \\
\hline Age (years) & $64(29,90)$ & $73(25,94)$ & $<0.0001$ \\
\hline Gender, male/female & $139 / 166$ & $26 / 25$ & 0.5446 \\
\hline $\mathrm{HBV} / \mathrm{HCV} / \mathrm{HBV}$ and $\mathrm{HCV} / \mathrm{NBNC}$ & $56 / 151 / 8 / 90$ & $5 / 30 / 1 / 15$ & 0.4421 \\
\hline Body mass index $\left(\mathrm{kg} / \mathrm{m}^{2}\right)$ & $22.9(14.8,41.4)$ & $22.4(14.6,30.6)$ & 0.1246 \\
\hline Presence of LC, yes/no & $109 / 196$ & $28 / 23$ & 0.0124 \\
\hline Total bilirubin (mg/dL) & $0.8(0.3,5.1)$ & $0.9(0.2,3.8)$ & 0.9470 \\
\hline Serum albumin $(\mathrm{g} / \mathrm{dL})$ & $4.3(2.0,5.1)$ & $4.1(2.4,5.2)$ & 0.0011 \\
\hline Prothrombin time (\%) & $91.4(11.9,122.9)$ & $88.9(33.4,122.5)$ & 0.1818 \\
\hline Platelet count $\left(\times 10^{4} / \mathrm{mm}^{3}\right)$ & $17.9(1.4,51.4)$ & $16.9(2.8,34.5)$ & 0.1531 \\
\hline AST & $25(12,222)$ & $24(10,191)$ & 0.3413 \\
\hline ALT & $20(6,206)$ & $17(5,232)$ & 0.6215 \\
\hline M2BPGi & $1.2(0.21,11.93)$ & $2.18(0.47,10.82)$ & 0.0016 \\
\hline eGFR $\left(\mathrm{ml} / \mathrm{min} / 1.73 \mathrm{~m}^{2}\right)$ & $81(5,173)$ & $73(7,162)$ & 0.0709 \\
\hline HbA1c (NGSP) & $5.7(3.7,12.6)$ & $5.7(4.5,9.8)$ & 0.5730 \\
\hline Serum ammonia $(\mu \mathrm{g} / \mathrm{dL})$ & $38(12,195)$ & $40(14,206)$ & 0.7299 \\
\hline BTR & $5.715(1.93,13.82)$ & $5.07(1.69,10.0)$ & 0.0289 \\
\hline Serum zinc $(\mu \mathrm{g} / \mathrm{dL})$ & $74.2(22.0,124.6)$ & $69.5(36.6,118.2)$ & 0.1474 \\
\hline ECW to TBW ratio & $0.389(0.367,0.425)$ & $0.398(0.368,0.421)$ & $<0.0001$ \\
\hline SMI decline, yes/no/unknown & $94 / 209 / 2$ & $27 / 24 / 0$ & 0.0037 \\
\hline
\end{tabular}

Continuous data are expressed as median (range). HCV, hepatitis C virus; HBV, hepatitis B virus; NBNC, non-B and non-C; LC, liver cirrhosis; AST, aspartate aminotransferase; ALT, alanine aminotransferase; M2BPGi, Mac-2 binding protein glycosylation isomer; eGFR, estimated glomerular filtration rate; NGSP, National Glycohemoglobin Standardization Program; BTR, branched-chain amino acid to tyrosine ratio; ECW, extracellular water; TBW, total body water; SMI, skeletal muscle index. 
Table 6. Multivariate analyses of factors linked to walking speed decline $(<1.0 \mathrm{~m} / \mathrm{s})$.

\begin{tabular}{cccc}
\hline & \multicolumn{3}{c}{ Multivariate Analysis } \\
\cline { 2 - 4 } & HR & $\mathbf{9 5 \% ~ C I ~}$ & $p$-Value \\
\hline Age \# & 0.0638 & $0.00205-1.989$ & 0.0985 \\
Presence of LC & 0.973 & $0.320-2.957$ & 0.9620 \\
SMI decline & 0.606 & $0.229-1.603$ & 0.3132 \\
Serum albumin $\#$ & 2.596 & $0.0989-6.817$ & 0.5665 \\
M2BPGi ${ }^{\#}$ & 0.887 & $0.0676-11.645$ & 0.9275 \\
BTR $^{\#}$ & 1.121 & $0.0760-16.540$ & 0.9337 \\
ECW to TBW ratio \# $^{*}$ & 0.0284 & $0.000715-1.132$ & 0.0556 \\
\hline
\end{tabular}

\# When a continuous variable changes over the entire range. HR, hazard ratio; CI, confidence interval; LC, liver cirrhosis; SMI, skeletal muscle index; M2BPGi, Mac-2 binding protein glycosylation isomer; BTR, branched-chain amino acid to tyrosine ratio; ECW, extracellular water; TBW, total body water.

\subsection{WS Stratified by ECW to TBW Ratio}

Based on the results of multivariate analyses, we further examined the impact of the ECW to TBW ratio on WS decline. Extracellular fluid excess was categorized as the following three types: normal condition (ECW to TBW ratio $<0.390, \mathrm{n}=178$ ), mild overhydrated condition (ECW to TBW ratio $0.390-0.399, \mathrm{n}=118$ ) and moderate to severe overhydrated condition (ECW to TBW ratio $\geq 0.400$, $\mathrm{n}=58$ ) [29]. As shown in Figure 7, the WS value was well stratified according to the ECW to TBW ratio ( $p$-values: normal vs. mild, $p=0.0001$; mild vs. moderate to severe, $p<0.0001$; normal vs. moderate to severe, $p<0.0001$; overall $p$-value $<0.0001$ ).

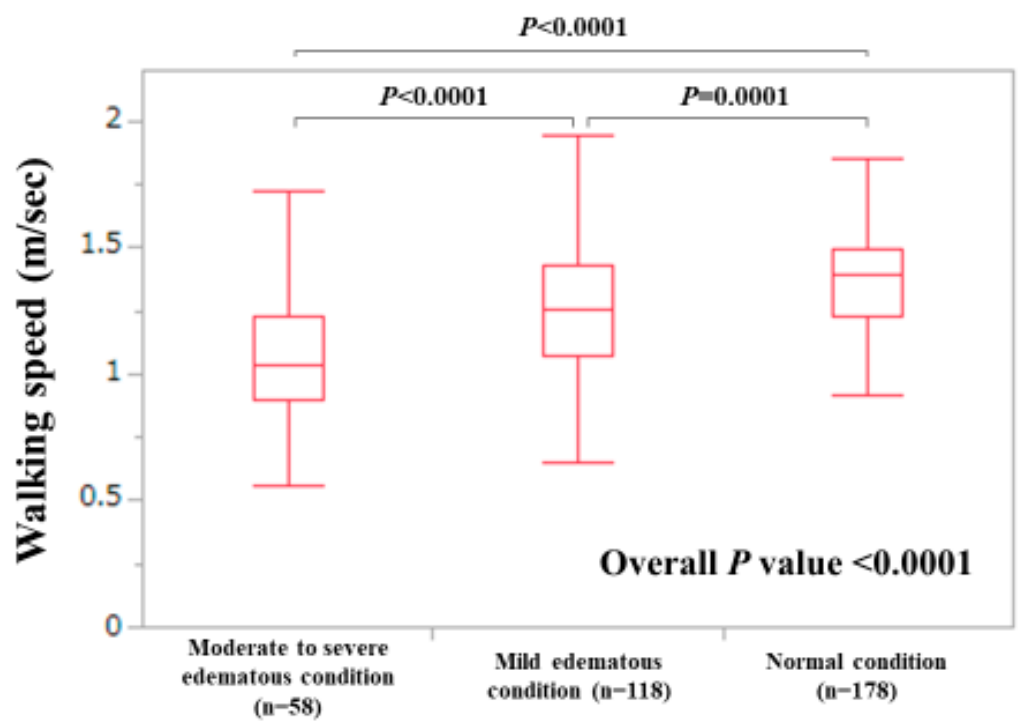

Figure 7. The WS value according to extracellular water (ECW) to total body water (TBW) as assessed by bioimpedance analysis. Normal condition indicates an ECW to TBW ratio $<0.390(\mathrm{n}=178$ ), mild overhydrated condition indicates an ECW to TBW ratio $0.390-0.399(n=118)$ and moderate to severe overhydrated condition indicates an ECW to TBW ratio $\geq 0.400(n=58)$. Missing data for the ECW to TBW ratio, $\mathrm{n}=2$.

\section{Discussion}

Physical activity has been considered a factor of interest in recent research. Previous data indicate that functional measures of muscle strength may have better predictability for outcomes than CT-based measures of skeletal muscle mass in LC patients [30]. As mentioned earlier, the current JSH guidelines for sarcopenia in CLDs adopt not WS but only HGS for the assessment of muscle strength decline and few research papers report data for WS in Japanese CLD patients $[10,18,19]$. Several reports have 
demonstrated that physical function precedes cognitive decline; however, the effects of HGS decline and WS decline on cognitive decline in adults may be different [20,31-33]. We, therefore, believe that to elucidate factors related to WS decline may be clinically meaningful.

In our data, the median (range) WS was $1.3 \mathrm{~m} / \mathrm{s}(0.2-2.02 \mathrm{~m} / \mathrm{s})$ and there were only 17 patients with WS $<0.8 \mathrm{~m} / \mathrm{s}(4.8 \%)$, which were in line with previous Japanese data [21]. In other words, as we expected, a small proportion of our study subjects had WS decline. In order to demonstrate the usefulness as a screening tool of WS for muscle strength decline, the cutoff value of WS $<1.0 \mathrm{~m} / \mathrm{s}$ can be considered, as recommended by the NILS-LSA and IWGS, while the significant difference of the WS values between elderly and younger patients and that between LC and non-LC patients well reflected primary and secondary sarcopenia in CLDs [10].

The WS value was significantly correlated with the HGS value both in males and females for all cases, LC patients and non-LC patients, in this study. However, there are some cases where there was a gap between the WS value and the HGS value, as shown in Figure 3A,B. The improvement in WS requires quick movement, whereas the improvement in the HGS does not require it and this may account for our current results. Both maintained cognitive function and muscle strength may be necessary for the improvement of WS, while even if cognitive function is reduced, the HGS value will not decrease as long as muscle strength is maintained [20,34]. While the correlation coefficient between the WS value and SMI was not impressive compared with that between the WS value and the HGS value, muscle function and muscle mass may play different roles for the development of sarcopenia $[19,30]$.

The ECW to TBW ratio is an independent factor linked to the WS decline $(<0.8 \mathrm{~m} / \mathrm{s})$ and tends to be a significant factor linked to WS decline $(<1.0 \mathrm{~m} / \mathrm{s})$ in our multivariate analysis. In addition, the WS value was well stratified according to the ECW to TBW ratio (normal condition, mild edematous condition, and moderate to severe edematous condition). The ECW to TBW ratio suggests extracellular fluid status (water homeostasis), including the whole body [29]. Excessive extracellular fluid may lead to the cognitive decline, and subsequent WS decline, which may be linked to our results $[35,36]$.

In our previous investigation, we found that M2BPGi, a novel liver fibrosis marker, significantly correlated with the HGS value in both males $(r=-0.4611, p<0.0001)$ and in females $(r=-0.33326$, $p<0.0001$ [37]. While in this study, the correlation coefficient between the WS value and M2BPGi was $-0.2555(p<0.0001)$, which was slightly different from that between HGS and M2BPGi. In view of these results, the HGS and the WS may not be the same for the evaluation of muscle strength. However, future investigations will be necessary to confirm these results.

Frailty as well as sarcopenia has received increasing scientific attention as a potential explanation of the health diversity of elderly persons these days [38]. Frailty indicates a state in which physical activities have deteriorated due to age [39]. Decreased HGS, decreased WS, and decreased SMI can be associated with frailty [40]. In this study, the WS value was significantly correlated with the HGS value both in males and females and such tendencies were not observed in the correlation between WS and SMI. However, whether WS is more a sign of frailty or sarcopenia than HGS or SMI in CLD patients is unclear. Further research will be required.

Several limitations related to this study warrant mention. First, this was a single-center observational study with retrospective properties. Second, the study data was derived from population data on liver diseases in Japan, and additional studies on other races are needed to further validate and extend its application to other races. Third, the WS value can vary depending on various physical conditions. Fourth, large ascites patients who may be involved in WS decline were excluded because of the limitation of BIA, being the possibility of bias. Fifth, the effects of several diseases other than liver diseases that can affect WS (e.g., orthopedic diseases) were not fully examined. Finally, because of the cross-sectional nature of the data, the direction of association between baseline data and WS remains unknown. The results, therefore, must be interpreted with caution. Nevertheless, our study results indicate that the WS value in CLDs is well correlated with age or liver functional markers. Especially, 
an edematous state, as evaluated by BIA (i.e., the ECW to TBW ratio), was closely linked to WS decline. The HGS and WS should not be considered as the same assessment tool for muscle strength.

Author Contributions: Data curation: H.N., K.Y., Y.I., Y.S., K.K., N.I. (Naoto Ikeda), T.T., N.A., R.T., K.H., N.I. (Noriko Ishii), Y.Y., T.N. and H.I.; Formal analysis: H.N.; Supervision: S.N.; Writing-original draft: H.N.; Writing-review \& editing: H.N., H.E. and S.N. All authors have read and agreed to the published version of the manuscript.

Funding: This research received no external funding.

Acknowledgments: The authors would like to thank all medical staff in our hospital for their support. This work was partly granted by Hyogo Innovative Challenge, Hyogo college of medicine, Japan.

Conflicts of Interest: The authors declare no conflicts of interest.

\section{Abbreviations}

$\begin{array}{ll}\text { CLD } & \text { chronic liver disease } \\ \text { LC } & \text { liver cirrhosis } \\ \text { BIA } & \text { bioimpedance analysis } \\ \text { CT } & \text { computed tomography } \\ \text { HGS } & \text { hand grip strength } \\ \text { WS } & \text { walking speed } \\ \text { EWGSOP } & \text { European Working Group on Sarcopenia in Older People } \\ \text { AWGS } & \text { Asian Working Group on Sarcopenia } \\ \text { JSH } & \text { Japanese society of hepatology } \\ \text { M2BPGi } & \text { Mac-2 binding protein glycosylation isomer } \\ \text { SMI } & \text { skeletal muscle index } \\ \text { IRB } & \text { institutional review board } \\ \text { m/s } & \text { meter/second } \\ \text { ECW } & \text { extracellular water } \\ \text { TBW } & \text { total body water } \\ \text { BTR } & \text { branched-chain amino acid to tyrosine ratio } \\ \text { HR } & \text { hazard ratio } \\ \text { CI } & \text { confidence interval } \\ \text { NILS-LSA } & \text { National Institute for Longevity Sciences-Longitudinal Study of Aging } \\ \text { IWGS } & \text { International Working Group on Sarcopenia }\end{array}$

\section{References}

1. Nishikawa, H.; Enomoto, H.; Yoh, K.; Iwata, Y.; Sakai, Y.; Kishino, K.; Ikeda, N.; Takashima, T.; Aizawa, N.; Takata, R.; et al. Combined Albumin-Bilirubin Grade and Skeletal Muscle Mass as a Predictor in Liver Cirrhosis. J. Clin. Med. 2019, 8, 782. [CrossRef] [PubMed]

2. Nishikawa, H.; Enomoto, H.; Yoh, K.; Iwata, Y.; Sakai, Y.; Kishino, K.; Ikeda, N.; Takashima, T.; Aizawa, N.; Takata, R.; et al. Health-Related Quality of Life in Chronic Liver Diseases: A Strong Impact of Hand Grip Strength. J. Clin. Med. 2018, 7, 553. [CrossRef] [PubMed]

3. Aby, E.S.; Saab, S. Frailty, Sarcopenia, and Malnutrition in Cirrhotic Patients. Clin. Liver Dis. 2019, 23, 589-605. [CrossRef] [PubMed]

4. Williams, F.R.; Berzigotti, A.; Lord, J.M.; Lai, J.C.; Armstrong, M.J. Review article: Impact of exercise on physical frailty in patients with chronic liver disease. Aliment. Pharmacol. Ther. 2019. [CrossRef] [PubMed]

5. Ebadi, M.; Bhanji, R.A.; Mazurak, V.C.; Montano-Loza, A.J. Sarcopenia in cirrhosis: From pathogenesis to interventions. J. Gastroenterol. 2019, 54, 845-859. [CrossRef]

6. Ooi, P.H.; Hager, A.; Mazurak, V.C.; Dajani, K.; Bhargava, R.; Gilmour, S.M.; Mager, D.R. Sarcopenia in Chronic Liver Disease: Impact on Outcomes. Liver Transpl. 2019, 25, 1422-1438. [CrossRef]

7. Hsu, C.S.; Kao, J.H. Sarcopenia and chronic liver diseases. Expert Rev. Gastroenterol. Hepatol. 2018, 12, 1229-1244. [CrossRef] 
8. Nardelli, S.; Gioia, S.; Faccioli, J.; Riggio, O.; Ridola, L. Sarcopenia and cognitive impairment in liver cirrhosis: A viewpoint on the clinical impact of minimal hepatic encephalopathy. World J. Gastroenterol. 2019, 25, 5257-5265. [CrossRef]

9. Wijarnpreecha, K.; Werlang, M.; Panjawatanan, P.; Kroner, P.T.; Cheungpasitporn, W.; Lukens, F.J.; Pungpapong, S.; Ungprasert, P. Association between sarcopenia and hepatic encephalopathy: A systematic review and meta-analysis. Ann. Hepatol. 2019, S1665-S2681. [CrossRef]

10. Nishikawa, H.; Shiraki, M.; Hiramatsu, A.; Moriya, K.; Hino, K.; Nishiguchi, S. Japan Society of Hepatology guidelines for sarcopenia in liver disease (1st edition): Recommendation from the working group for creation of sarcopenia assessment criteria. Hepatol. Res. 2016, 46, 951-963. [CrossRef]

11. Arai, H.; Akishita, M.; Chen, L.K. Growing research on sarcopenia in Asia. Geriatr. Gerontol. Int. 2014, 14, 1-7. [CrossRef] [PubMed]

12. Sinclair, M.; Gow, P.J.; Grossmann, M.; Angus, P.W. Review article: Sarcopenia in cirrhosis-Aetiology, implications and potential therapeutic interventions. Aliment. Pharmacol. Ther. 2016, 43, 765-777. [CrossRef]

13. Lai, J.C.; Covinsky, K.E.; McCulloch, C.E.; Feng, S. The Liver Frailty Index Improves Mortality Prediction of the Subjective Clinician Assessment in Patients with Cirrhosis. Am. J. Gastroenterol. 2018, 113, $235-242$. [CrossRef] [PubMed]

14. Bhanji, R.A.; Montano-Loza, A.J.; Watt, K.D. SARCOPENIA IN CIRRHOSIS: Looking beyond the skeletal muscle loss to see the systemic disease. Hepatology 2019. [CrossRef] [PubMed]

15. Cruz-Jentoft, A.J.; Bahat, G.; Bauer, J.; Boirie, Y.; Bruyère, O.; Cederholm, T.; Cooper, C.; Landi, F.; Rolland, Y.; Sayer, A.A.; et al. Writing Group for the European Working Group on Sarcopenia in Older People 2 (EWGSOP2), and the Extended Group for EWGSOP2. Sarcopenia: Revised European consensus on definition and diagnosis. Age Ageing 2019, 48, 16-31. [CrossRef] [PubMed]

16. Chen, L.K.; Liu, L.K.; Woo, J.; Assantachai, P.; Auyeung, T.W.; Bahyah, K.S.; Chou, M.Y.; Chen, L.Y.; Hsu, P.S.; Krairit, O.; et al. Sarcopenia in Asia: Consensus report of the Asian Working Group for Sarcopenia. J. Am. Med. Dir. Assoc. 2014, 15, 95-101. [CrossRef]

17. Fielding, R.A.; Vellas, B.; Evans, W.J.; Bhasin, S.; Morley, J.E.; Newman, A.B.; van Kan, G.A.; Andrieu, S.; Bauer, J.; Breuille, D.; et al. Sarcopenia: An undiagnosed condition in older adults. Current consensus definition: Prevalence, etiology, and consequences. International working group on sarcopenia. J. Am. Med. Dir. Assoc. 2011, 12, 249-256. [CrossRef]

18. Nagamatsu, A.; Kawaguchi, T.; Hirota, K.; Koya, S.; Tomita, M.; Hashida, R.; Kida, Y.; Narao, H.; Manako, Y.; Tanaka, D.; et al. Slow walking speed overlapped with low handgrip strength in chronic liver disease patients with hepatocellular carcinoma. Hepatol. Res. 2019. [CrossRef]

19. Harimoto, N.; Yoshizumi, T.; Izumi, T.; Motomura, T.; Harada, N.; Itoh, S.; Ikegami, T.; Uchiyama, H.; Soejima, Y.; Nishie, A.; et al. Clinical Outcomes of Living Liver Transplantation According to the Presence of Sarcopenia as Defined by Skeletal Muscle Mass, Hand Grip, and Gait Speed. Transplant. Proc. 2017, 49, 2144-2152. [CrossRef]

20. Chou, M.Y.; Nishita, Y.; Nakagawa, T.; Tange, C.; Tomida, M.; Shimokata, H.; Otsuka, R.; Chen, L.K.; Arai, H. Role of gait speed and grip strength in predicting 10-year cognitive decline among community-dwelling older people. BMC Geriatr. 2019, 19, 186. [CrossRef]

21. Yoshida, D.; Suzuki, T.; Shimada, H.; Park, H.; Makizako, H.; Doi, T.; Anan, Y.; Tsutsumimoto, K.; Uemura, K.; Ito, T.; et al. Using two different algorithms to determine the prevalence of sarcopenia. Geriatr. Gerontol. Int. 2014, 14, 46-51. [CrossRef] [PubMed]

22. Fukui, H.; Saito, H.; Ueno, Y.; Uto, H.; Obara, K.; Sakaida, I.; Shibuya, A.; Seike, M.; Nagoshi, S.; Segawa, M.; et al. Evidence-based clinical practice guidelines for liver cirrhosis 2015. J. Gastroenterol. 2016, 51, 629-650. [CrossRef] [PubMed]

23. Kudo, M.; Zheng, R.Q.; Kim, S.R.; Okabe, Y.; Osaki, Y.; Iijima, H.; Itani, T.; Kasugai, H.; Kanematsu, M.; Ito, K.; et al. Diagnostic accuracy of imaging for liver cirrhosis compared to histologically proven liver cirrhosis. A multicenter collaborative study. Intervirology 2008, 51, 17-26. [CrossRef] [PubMed]

24. Zarski, J.P.; Sturm, N.; Guechot, J.; Paris, A.; Zafrani, E.S.; Asselah, T.; Boisson, R.C.; Bosson, J.L.; Guyader, D.; Renversez, J.C.; et al. ANRS HCEP 23 Fibrostar Group. Comparison of nine blood tests and transient elastography for liver fibrosis in chronic hepatitis C: The ANRS HCEP-23 study. J. Hepatol. 2012, 56, 55-62. [CrossRef] 
25. Tsochatzis, E.A.; Gurusamy, K.S.; Ntaoula, S.; Cholongitas, E.; Davidson, B.R.; Burroughs, A.K. Elastography for the diagnosis of severity of fibrosis in chronic liver disease: A meta-analysis of diagnostic accuracy. J. Hepatol. 2011, 54, 650-659. [CrossRef]

26. Hasegawa, K.; Takata, R.; Nishikawa, H.; Enomoto, H.; Ishii, A.; Iwata, Y.; Miyamoto, Y.; Ishii, N.; Yuri, Y.; Nakano, C.; et al. Impact of Wisteria floribunda Agglutinin-Positive Mac-2-Binding Protein in Patients with Hepatitis C Virus-Related Compensated Liver Cirrhosis. Int. J. Mol. Sci. 2016, 17, 1500. [CrossRef]

27. Nishikawa, H.; Enomoto, H.; Ishii, A.; Iwata, Y.; Miyamoto, Y.; Ishii, N.; Yuri, Y.; Takata, R.; Hasegawa, K.; Nakano, C.; et al. Development of a simple predictive model for decreased skeletal muscle mass in patients with compensated chronic liver disease. Hepatol. Res. 2017, 47, 1223-1234. [CrossRef]

28. Shimokata, H.; Ando, F.; Niino, N. A new comprehensive study on aging-the National Institute for Longevity Sciences, Longitudinal Study of Aging (NILS-LSA). J. Epidemiol. 2000, 10, S1-S9. [CrossRef]

29. Nishikawa, H.; Yoh, K.; Enomoto, H.; Ishii, N.; Iwata, Y.; Nakano, C.; Takata, R.; Nishimura, T.; Aizawa, N.; Sakai, Y.; et al. Extracellular Water to Total Body Water Ratio in Viral Liver Diseases: A Study Using Bioimpedance Analysis. Nutrients 2018, 10, 1072. [CrossRef]

30. Wang, C.W.; Feng, S.; Covinsky, K.E.; Hayssen, H.; Zhou, L.Q.; Yeh, B.M.; Lai, J.C. A Comparison of Muscle Function, Mass, and Quality in Liver Transplant Candidates: Results from the Functional Assessment in Liver Transplantation Study. Transplantation 2016, 100, 1692-1698. [CrossRef]

31. Sternäng, O.; Reynolds, C.A.; Finkel, D.; Ernsth-Bravell, M.; Pedersen, N.L.; Dahl Aslan, A.K. Grip strength and cognitive abilities: Associations in old age. J. Gerontol. B Psychol. Sci. Soc. Sci. 2015, 71, 841-848. [CrossRef] [PubMed]

32. Inzitari, M.; Newman, A.B.; Yaffe, K.; Boudreau, R.; de Rekeneire, N.; Shorr, R.; Harris, T.B.; Rosano, C. Gait speed predicts decline in attention and psychomotor speed in older adults: The health aging and body composition study. Neuroepidemiology 2007, 29, 156-162. [CrossRef] [PubMed]

33. Hsu, C.L.; Liang, C.K.; Liao, M.C.; Chou, M.Y.; Lin, Y.T. Slow gait speed as a predictor of 1-year cognitive decline in a veterans' retirement community in southern Taiwan. Geriatr. Gerontol. Int. 2017, 17, 14-19. [CrossRef] [PubMed]

34. Clouston, S.A.; Brewster, P.; Kuh, D.; Richards, M.; Cooper, R.; Hardy, R.; Rubin, M.S.; Hofer, S.M. The dynamic relationship between physical function and cognition in longitudinal aging cohorts. Epidemiol. Rev. 2013, 35, 33-50. [CrossRef]

35. Hadjihambi, A.; Arias, N.; Sheikh, M.; Jalan, R. Hepatic encephalopathy: A critical current review. Hepatol. Int. 2018, 12, 135-147. [CrossRef]

36. Ji, F.; Pasternak, O.; Liu, S.; Loke, Y.M.; Choo, B.L.; Hilal, S.; Xu, X.; Ikram, M.K.; Venketasubramanian, N.; Chen, C.L.; et al. Distinct white matter microstructural abnormalities and extracellular water increases relate to cognitive impairment in Alzheimer's disease with and without cerebrovascular disease. Alzheimers Res. Ther. 2017, 9, 63. [CrossRef]

37. Nishikawa, H.; Enomoto, H.; Yoh, K.; Iwata, Y.; Sakai, Y.; Kishino, K.; Ikeda, N.; Takashima, T.; Aizawa, N.; Takata, R.; et al. Significant Correlation Between Grip Strength and m2bpgi in Patients with Chronic Liver Diseases. J. Clin. Med. 2019, 8, 1359. [CrossRef]

38. Kojima, G.; Liljas, A.E.M.; Iliffe, S. Frailty syndrome: Implications and challenges for health care policy. Risk Manag. Healthc. Policy 2019, 12, 23-30. [CrossRef]

39. Waldon, M. Frailty in older people: A principle-based concept analysis. Br. J. Community Nurs. 2018, 23, 482-494. [CrossRef]

40. Cruz-Jentoft, A.J.; Kiesswetter, E.; Drey, M.; Sieber, C.C. Nutrition, frailty, and sarcopenia. Aging Clin. Exp. Res. 2017, 29, 43-48. [CrossRef]

(C) 2020 by the authors. Licensee MDPI, Basel, Switzerland. This article is an open access article distributed under the terms and conditions of the Creative Commons Attribution (CC BY) license (http://creativecommons.org/licenses/by/4.0/). 